\title{
A multi-scale study of residual stresses created during the cure process of a composite tooling material
}

\author{
E. Lacoste ${ }^{1, a}$, S. Fréour ${ }^{1, b}$, F. Jacquemin ${ }^{1, c}$ \\ ${ }^{1}$ Institut de Recherche en Génie Civil et Mécanique (UMR CNRS 6183), Université de Nantes - \\ Centrale Nantes, 37 Boulevard de l'Université, BP 406, 44602 Saint-Nazaire cedex, France \\ a emmanuel.lacoste@univ-nantes.fr, ${ }^{\mathrm{b}}$ sylvain.freour@univ-nantes.fr, \\ c frederic.jacquemin@univ-nantes.fr
}

KeyWords: Composite materials, cure process, residual stresses, scale transition model.

\begin{abstract}
This paper deals with the cure of an in-plane isotropic carbon-polymer tooling material, with a complex microstructure [1]. The Mori-Tanaka (MT) and Eshelby-Kröner self-consistent (EKSC) models are used in order to achieve a two-steps scale transition procedure, relating the microscopic properties of the material to their macroscopic counterparts. This procedure enables estimating the multi-scale mechanical states experienced by the material, i.e. the local (microscopic) stresses due to thermal and chemical shrinkage of the resin, along a typical, macroscopic stress-free, cure process. The influence of the chosen scale transition model on both the calculated effective properties of the material and its local stress states, is investigated. These results are a first step for investigating the service life fatigue of the material, as well as its failure behaviour.
\end{abstract}

\section{Introduction}

Context. In the last decade, composite materials based on carbon (or glass) fibers and thermoset resins have been more and more involved in the design of mechanical parts. However, the engineering of composite parts yields several scientific challenges. One of them is the computation of the microscopic internal stresses, due to the properties mismatch between the fibers and the matrix. As curing is considered, the strong thermo-chemical shrinkage of the matrix is counteracted by the fibers, and yield self-compensated stresses in the constituents. These stresses can significantly affect the macroscopic stress-strain, fatigue and failure behaviour, and can result in fiber-matrix debonding or in increased fibers waviness (microbuckling) [2,3].

Review of predictive models. Various experimental approaches were developed for measuring these local stresses and strains; an extensive summary of these methods is given by Parlevliet et al. [4]. Meanwhile, scale transition models proved to be a relevant predictive approach. Tsaï-Hahn's rules of mixtures [5] first gave an easily-understandable estimate of the properties of unidirectional composite plies, taking into account the anisotropy, but failed to predict the local stress states. Another class of models, the so-called "self-consistent" models, became trendier in the last decade. Among them, Mori-Tanaka (MT) [6] and Eshelby-Kröner Self-Consistent (EKSC) estimates [7,8] are the most used. These models can take into account the morphologies of the heterogeneities (inclusions) constituting the material $[9,10]$. They can also systematically predict the distribution of the mechanical states in the very constituents of the materials. It was recently taken advantage of this capability in order to realistically model realistically the various features of the multi-scale hygro-mechanical coupled phenomena occurring in composite structures submitted to humid air $[11,12]$.

Purpose of the study. The study is focused on a composite tooling material with a non-trivial microstructure, which cannot properly be accounted for, by classical rules of mixture. The EKSC and MT models will be used to carry out a multi-scale analysis of the material behaviour during the cure process. The evolutions of the properties of the resin during the cure will be described, and used to predict the effective mechanical behaviour of the material and the development of residual stresses. The influence of the chosen model on the results will then be investigated and discussed. 


\section{The scale transition procedure}

Description of the models. Scale transition models are based upon a representation of the material at two distinct scales: a) the local scale (denoted by the superscript ${ }^{\mathrm{i}}$ ), of the size of the constituents and b) the macroscopic scale $\left({ }^{\mathrm{I}}\right)$, where the behaviour of the effective medium is observed. The corresponding mechanical states are related through Hill's volume weighted average relations [13]:

$$
\varepsilon^{I}=\left\langle\varepsilon^{i}\right\rangle \text { and } \sigma^{I}=\left\langle\sigma^{i}\right\rangle .
$$

The arithmetic mean was used according to [14], which showed that it provides more reliable estimates than the geometric average for modeling organic matrix composites. In a previous work, Eshelby studied the behavior of an ellipsoidal inclusion embedded in a homogeneous ambient medium ${ }^{\text {a }}$ loaded at the infinite [15]. His work led to the following relation, expressed by Hill [16]:

$$
\sigma^{\mathrm{i}}-\sigma^{\mathrm{a}}=-\mathbf{L}^{*}:\left(\varepsilon^{\mathrm{i}}-\varepsilon^{\mathrm{a}}\right)
$$

where $\mathbf{L}^{*}$ represents Hill's constraint tensor, which depends upon the stiffness of the medium, and the morphology (orientation and shape factors) of the inclusion (see also $[8,17,18]$ ). Both relations, combined with the behaviour law, are used for estimating the effective (macroscopic) properties of the material. They also provide an estimate of the mean local mechanical states, with the following approximations indeed: the inter-particles interactions, the interphase and interfacial effects, are not represented. That's why the choice of the embedding medium has a deep influence on the predicted local states. This choice distinguishes the MT from the EKSC model. For the EKSC scheme, the embedding medium is supposed to have the properties of the effective material at the macroscopic scale; whereas for the MT model [6], one of the constituents (usually, the matrix phase or the dominant phase) is chosen as the ambient medium.

Application to the Hextool. The present work is focused on a composite material developed by Hexcel Composites for manufacturing complex parts: the Hextool [19]. It is made of unidirectional (UD) rectangular-shaped $(60 \times 8 \times 0.15 \mathrm{~mm})$ reinforcing strips, randomly disposed in the layout (Fig. 1b). The UD strips are constituted by AS4 fibers and M61C system (high-temperature toughened bismaleimid resin) [19]. The overall resin volume ratio is about $47 \%$, distributed inside the strips and between the strips ( $5 \%$ of the overall resin volume). Thereby, the structure of the material involves three different scales: microscopic, mesoscopic and macroscopic. A two-steps scale transition procedure (Fig. 1a) is performed: first, the properties of resin and fibers (microscopic scale) are homogenized to obtain those of the UD strip (mesoscopic scale). Then, another homogenization provides the macroscopic properties, from those of the resin and the UD strips (mesoscopic scale) previously estimated.

Figure 1: (a) Principle of the two-steps scale transition procedure

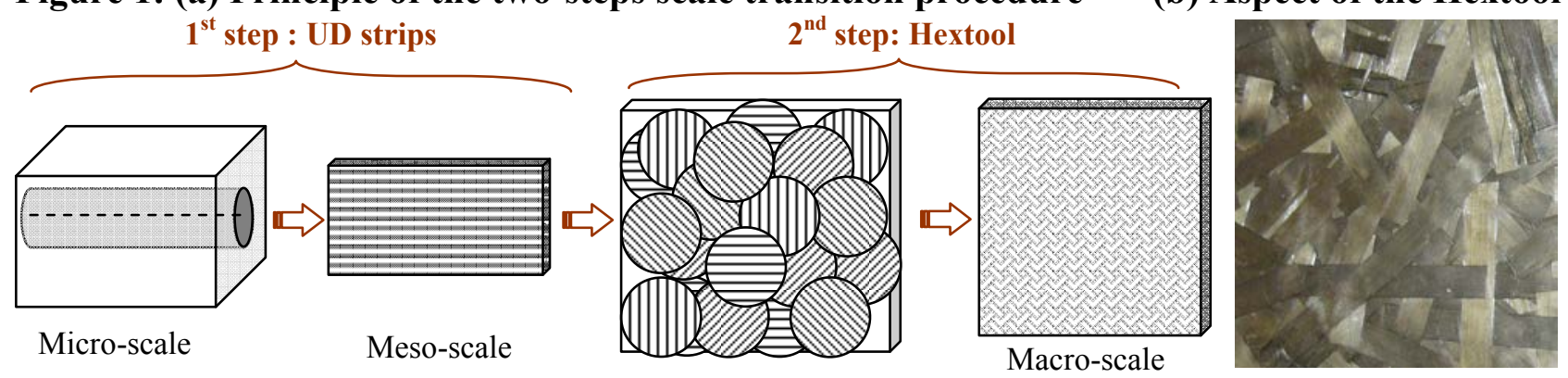

For the first step, three options will be considered: using MT model with either the resin (MTres) or the fibers (MTfib) as the embedding medium, and using the EKSC model (effective material as the embedding medium). For the second step, only EKSC model was considered, as one cannot distinguish any dominant phase. Moreover, the reinforcing strips were assumed to be disc-shaped for avoiding some limits of EKSC model [1] and optimizing computation time. 


\section{Investigation of the mechanical properties and cure kinetics of the resin}

During the cure process, the local and macroscopic behaviours can be described by the following differential chimio-thermo-elastic law, relating the stress $\boldsymbol{\sigma}$ to the strain $\boldsymbol{\varepsilon}$ :

$$
\dot{\sigma}^{\mathbf{k}}=\mathbf{L}^{\mathbf{k}}:\left(\dot{\varepsilon}^{\mathbf{k}}-\alpha^{\mathbf{k}} \dot{\mathrm{T}}-\eta^{\mathbf{k}} \dot{\chi}\right) \text { with } \mathrm{k}=\{\mathrm{i}, \mathrm{I}\},
$$

where the stiffness is represented by the $4^{\text {th }}$-order tensor $\mathbf{L}$ and the Coefficients of Thermal Expansion (CTE) and Chemical Expansion (CCE) by the $2^{\text {nd }}$-order tensors $\boldsymbol{\alpha}$ and $\boldsymbol{\eta}$, respectively. The temperature and conversion degree are denoted by $\mathrm{T}$ and $\chi$, and the time derivative by the upperscript ' A differential form is required, as the resin undergoes important evolutions of its properties during the cure, that strongly affect the development of residual stresses. In order to precisely describe those evolutions, the mechanical properties of the M61 resin were investigated.

Cure kinetics. The autocatalytic polymerisation reaction of the M61 resin can be parameterized by the conversion degree $\chi$, defined as the ratio of the enthalpy released at a time, per the overall released enthalpy. This conversion degree is a state variable, and can be used for identifying the mechanical properties of the resin. The cure kinetics of a M21 organic resin, obtained with Differential Scanning Calorimetry (DSC) by Msallem et al. [20] was used in the present study. A $180^{\circ} \mathrm{C}$ cure cycle was considered, as described in (Fig. 2a). The gradients of temperature and conversion degree, which can occur in thick parts due to the exothermic reaction, were neglected.

Figure 2: (a) Cure cycle and conversion degree

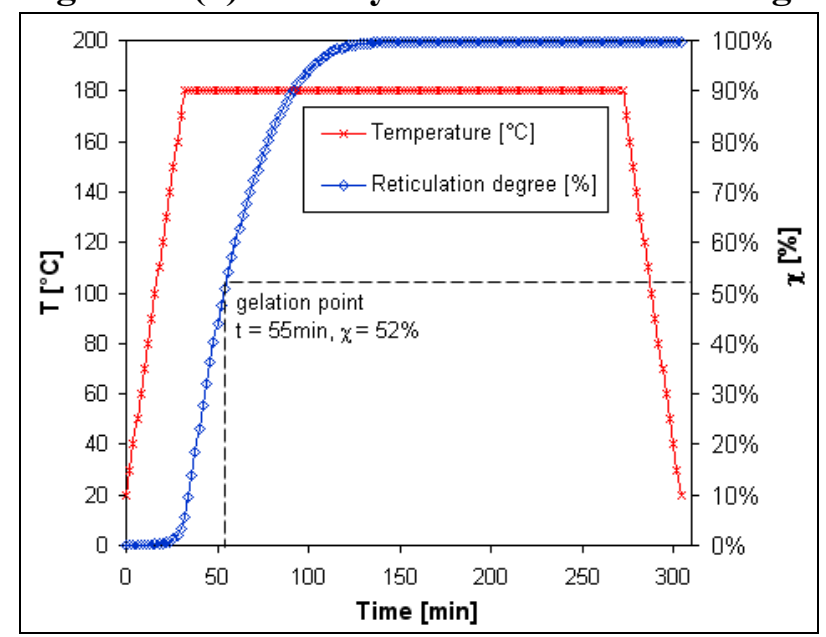

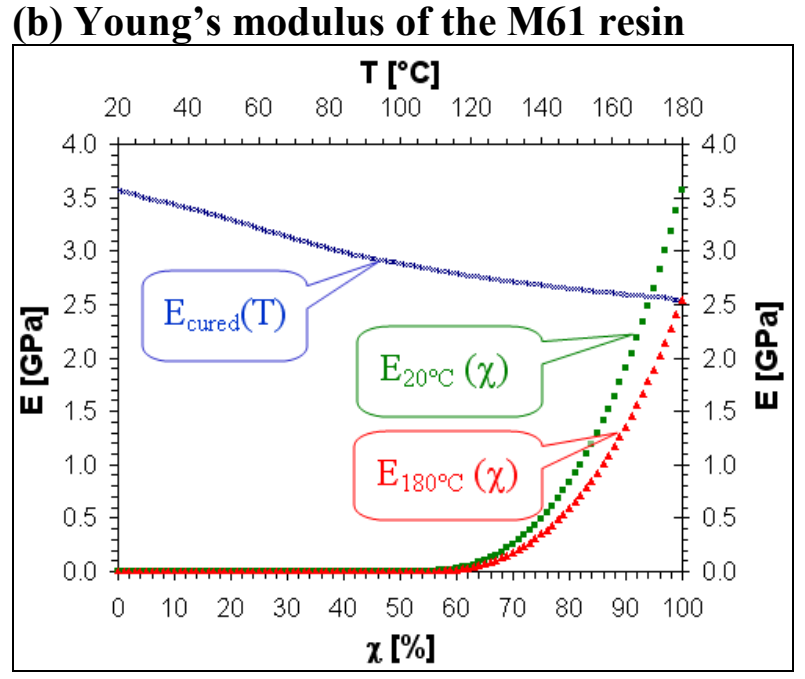

Elastic modulus. The extreme evolution of the viscoelastic modulus of the resin reflects the deep changes of its physical state. At the beginning of the cure, the resin is in a liquid state and has a viscous behaviour. At the gelation point, the resin turns into a solid state, and the relaxation times become important enough so that residual stresses start to develop. The stiffness then increases with the conversion. Although several models were developed to model this hardening of the resin, most authors assume a linear dependence of the elastic modulus upon the conversion degree. However, the percolation theory [21] gives a more suitable expression, as discussed in [20]:

$$
\mathrm{E}(\mathrm{T}, \chi)=\mathrm{E}_{\text {cured }}(\mathrm{T}) \cdot\left(\frac{\chi^{2}-\chi_{\text {gel }}^{2}}{1-\chi_{\text {gel }}^{2}}\right)^{8 / 3} \text {, }
$$

$\chi_{\text {gel }}$ stands for the conversion degree at the gelation point, whereas $E_{\text {cured }}(T)$ the elastic modulus of the fully cured resin. Fig. $2 \mathrm{~b}$ above displays the resulting evolution of the elastic modulus versus the temperature and reticulation degree. The evolution of the stiffness with temperature was determined, by co-workers (see acknowledgements), through Dynamic Mechanical Analysis (DMA). DMA provides the instantaneous modulus, which is very close to the equilibrium stiffness if the resin is in a vitreous state. This hypothesis might be partially wrong during the hardening 
phase, as the vitreous transition temperature is close to the cure temperature. The (Eq. 4) used here neglects the relaxation phenomena, and may thus yield a slight overestimation of local stresses.

Thermal expansion. The CTE of the resin also experiences severe evolutions during the curing process: it increases with the temperature and decreases with the conversion degree. However, the dependency on conversion degree should not influence the results here, as the resin is either liquid or fully cured during the heating and cooling stages of the cure cycle. The evolutions of the CTE during the cure process were described by (Eq. 5):

$$
\alpha(T, \chi)=\chi \times \alpha_{\text {cured }}(T)+(1-\chi) \times \alpha_{\text {raw }}(T) \text { with }\left\{\begin{array}{l}
\alpha_{\text {cured }}(T)=35.465+0.1191 \times T+0.0003 \times T^{2} \\
\alpha_{\text {raw }}(T)=4 \times \alpha_{\text {cured }}(T)
\end{array} .\right.
$$

Cure shrinkage. The polymerisation reaction corresponds to the formation of covalent bonds between the macromolecules and a constriction of the amorphous network. This induces important bulk shrinkage of the resin, in the order of $3 \%$ up to $9 \%$, which is comparable to the thermal shrinkage. In this study, we use a value of $5.7 \%$ for the chemical volume shrinkage [20], which corresponds to a linear Coefficient of Chemical Expansion $\eta$, equal to $-1.9 \%$.

\section{Results of the scale transition procedure}

Computation of effective properties. The scale transition procedure presented above is used to compute the effective properties of the material during the cure process, summed up in (Fig. 3). The gelation point is denoted by a marked gap of some properties. The choice of a model or another mainly affects the out-of-plane properties, but also the in-plane stiffness; the EKSC model results in a more rigid material than the MTres model, but less rigid than the MTfib model.

Figure 3: Evolutions of the main effective properties of the Hextool during the cure process

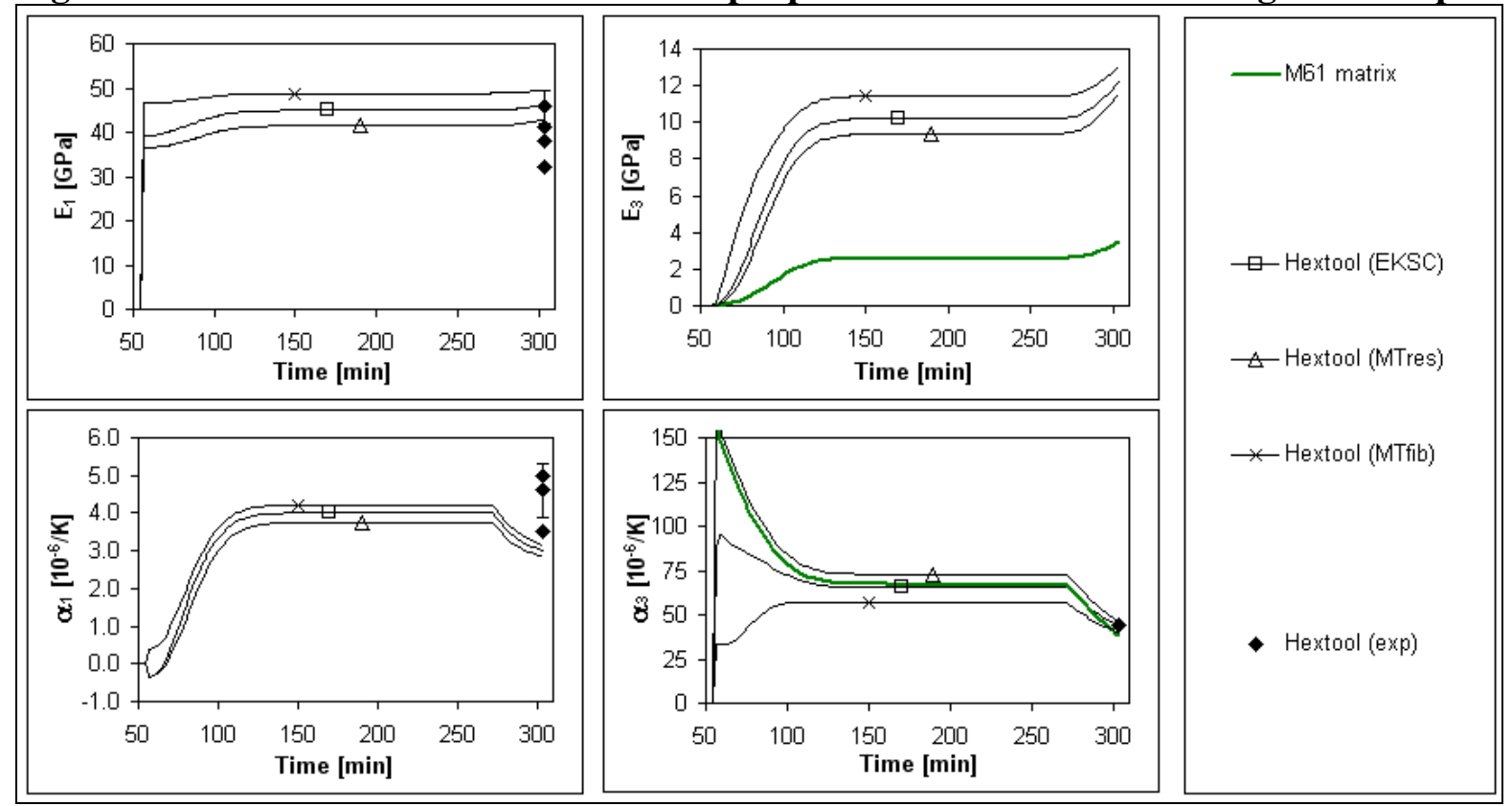

The few available experimental results [19] are consistent with the estimated effective properties, even though discrepancies occur for the in-plane properties (weaker stiffness and stronger coefficients of expansion). The discrepancies are attributed to out-of-plane waviness of the reinforcing strips, and also to the uncertainties on the fiber content.

Computation of local residual stresses. The scale transition procedure was used in order to incrementally compute the residual stresses along the cure process. The macroscopic stresses were supposed null, although other boundary conditions (fixed in-plane displacement, interactions with a metallic mold...) can be considered in the same way. Whatever, extensive tests showed that local stress created by those "external stresses" mostly vanish after removal from the mould. 
Figure 4: Residual cure stresses in the material

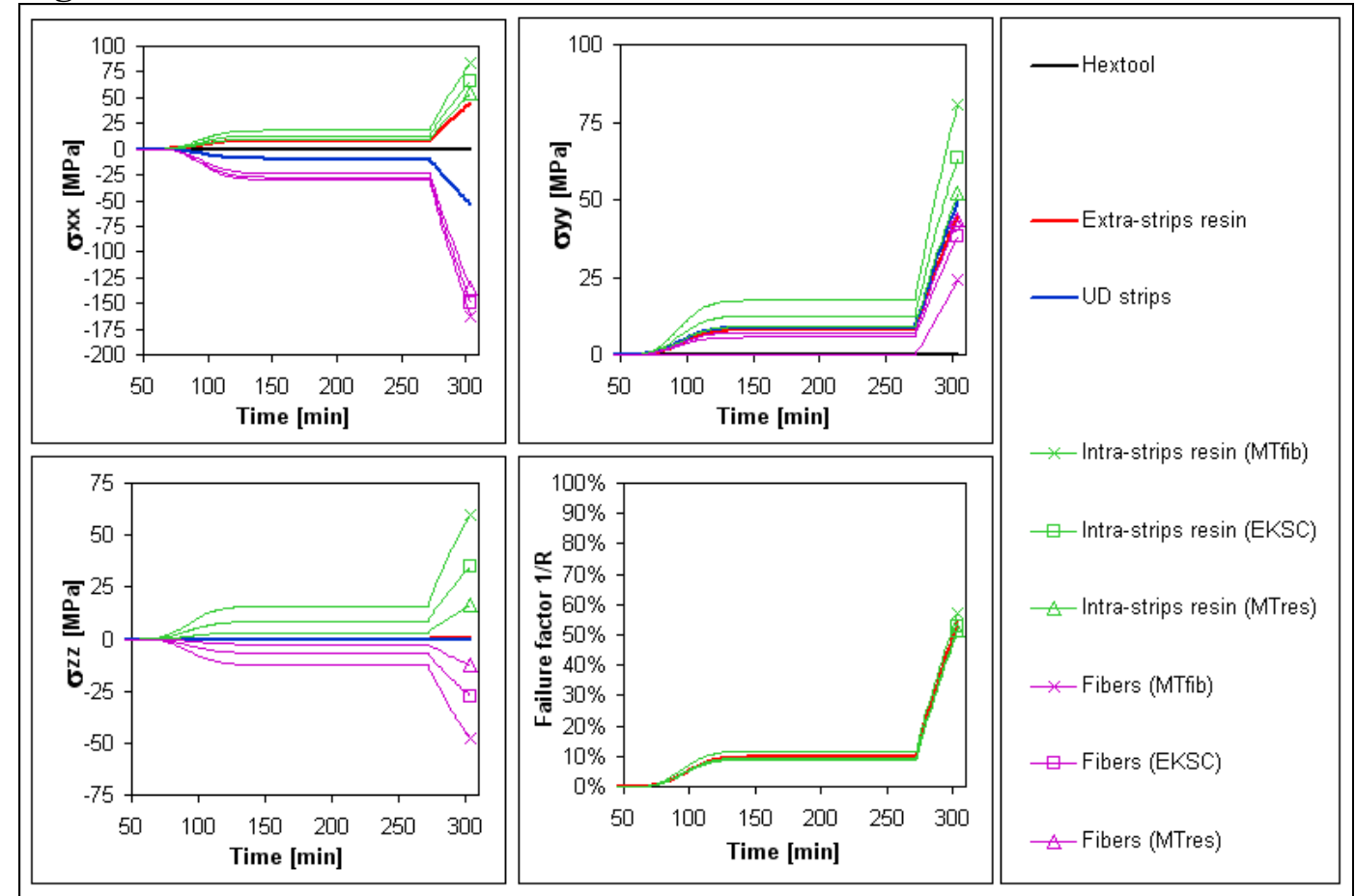

(Fig. 4) above shows the development of residual stresses at the macro, meso and micro levels, dropped in the coordinate system of each reinforcing strips ( $x, y$ and $z$ respectively stand for the axial, transverse and out-of-plane directions). One can observe three well-differentiated periods, corresponding to the pre-gelation phase, the hardening, and the thermal cooling. The largest part of residual stresses is created by thermal shrinkage. The role of chemical shrinkage is also significant, but may be overestimated, due to relaxation phenomena. Residual transverse stresses evolutions, predicted in the present study, can be qualitatively compared to those obtained by White and Kim [22] on a $\left[0^{\circ} / 90^{\circ}\right]_{\mathrm{s}}$ carbon-epoxy laminate subjected to manufacturing process. This comparison is limited by the Hextool's specific microstructure and resin (BMI instead of epoxy).

The choice of the model affects the residual stresses at the meso-level, only. Thus, MT and KESC estimates provide identical results for the UD strips and the extra-strips resin. At the microscopic scale (intra-strips resin and fibers), the MTfib model results in the strongest stresses and the MTres model in the weakest. This gap is particularly sensitive along $y$ and $z$ axes. In the x-direction, the rigid elements undergo compression stresses which may yield micro- and meso-bucklings phenomena. The wavy aspect of the Hextool ply seems to corroborate this statement. On the contrary, the resin undergoes important traction stresses in every direction, which could be the source of delaminations. A Tsaï-Wu quadratic failure criterion [5] enabled quantifying this risk, using data taken in the literature for a high-strength epoxy resin [23]. The values of the "failure factor" (inverse of the standard resistance R) are widely weaker than $100 \%$, which indicates that the residual stresses should not damage the material after the cure process.

\section{Conclusions and perspectives}

Two scale transition models were used for describing the multi-scale mechanical behaviour of a meso-structured composite material, through a two-steps procedure. This method was applied to the prediction of effective properties and local stress states in the composite, during the cure process. The results show significant residual stresses at the micro-level, but also the strong influence of the chosen scale transition model: the results given by the EKSC model, widely used for computing local stresses, can be enclosed by the two versions of the MT model. The thermal stresses were found predominant over those created by the chemical shrinkage, which may moreover be partially or totally relaxed. According to the results of a Tsaï-Wu failure criterion, the residual stresses in the 
resin should not induce material damage, but may play a significant role on failure under service loads. The method could also be applied for predicting the material service-life duration (fatigue under repeated thermal cycles, in particular). In further works, those estimates shall be compared to full-field approaches, which provide more realistic estimates of the local stress fields.

\section{Acknowledgements}

The authors wish to acknowledge C. Dauphin and M. Bonnafoux (from Hexcel Composites France) for the valuable information provided on the Hextool. We also wish to thank K. Szymanska, S. Terekhina and M. Salvia, from the Laboratoire de Tribologie et de Dynamique des Systèmes (LTDS) of Lyon (France), who provided the results of DMA performed on the M61 resin system.

\section{References}

[1] E. Lacoste, S. Fréour and F. Jacquemin: Mechanics of Materials Vol. 42 (2010), pp. 218-226.

[2] P.P. Parlevliet, H.E.N. Bersee, A. Beukers: Composites Part A Vol. 37 (2006), pp. 1847-1857.

[3] P.P. Parlevliet, H.E.N. Bersee, A. Beukers: Composites Part A Vol. 38 (2007), pp. 651-665.

[4] P.P. Parlevliet, H.E.N. Bersee, A. Beukers: Composites Part A Vol. 38 (2007), pp. 1581-1596

[5] S.W. Tsaï and H.T. Hahn, in: Introduction to composite materials, Tecnomic Publishing co., Inc., Lancaster, Pennsylvania (1980).

[6] T. Mori and K. Tanaka: Acta Metallurgica Vol. 21 (1973), pp. 571-574.

[7] J. Berryman and P. Berge: Mechanics of Materials Vol. 22 (1996), pp. 149-164.

[8] U. Kocks, C.N. Tomé and H.R. Wenk: Texture and anisotropy, Cambridge Univ. Press (1998).

[9] S. Fréour, F. Jacquemin and R. Guillén: J. Reinf. Plast. Comp. Vol. 24 (2005), pp. 1365-1377.

[10] S. Fréour, F. Jacquemin and R. Guillén: J. Reinf. Plast. Comp. Vol. 25 (2006), pp. 1039-1053.

[11] G. Youssef, S. Fréour and F. Jacquemin: J. Comp. Mat. Vol. 43 (2009), pp. 1621-1637.

[12] G. Youssef, S. Fréour and F. Jacquemin: Mech. Comp. Mat. Vol. 45 (2009), pp. 369-380.

[13] R. Hill: Journal of the Mechanics and Physics of Solids Vol. 15 (1967), pp. 79-95.

[14] S. Fréour, F. Jacquemin and R. Guillén: J. Mat. Sci., Vol. 42 (2007), pp. 7537-7543.

[15] J.D. Eshelby: Proceedings of the Royal Society London, A241 (1957), pp. 376-396.

[16] R. Hill: Journal of the Mechanics and Physics of Solids, Vol. 13 (1965), pp. 89-101.

[17]E. Kröner: Zeitschrift für Physik Vol. 151 (1958), pp. 504-508.

[18] T. Mura: Micromechanics of Defects in Solids (Martinus Nijhoff Pub., Netherlands, 1982).

[19] Information on http://www.hexcel.com/Products/Downloads/

[20]Y.A. Msallem, F. Jacquemin, N. Boyard, A. Poitou, D. Delaunay, S. Chatel: Composites Part A Vol. 41 (2010), pp. 108-115.

[21]D.B. Adolf and R.S. Chambers: Polymer Vol. 38 (1997), pp. 5481-5490.

[22] S.R. White and Y.K. Kim: Mech. of Comp. Mater. and Struct. Vol. 5 (1998), pp. 153-186.

[23]B. Fiedler, M. Hojo, S. Ochiai, K. Schulte and M. Ando: Composites Science and Technology Vol. 61 (2001), pp. 1615-1624. 